\title{
Automotive Paint Shop with Lowest Emissions in China
}

\author{
Dürr is building an automotive paint shop with the lowest emissions in China \\ for the FAW-VW joint venture. The paint shop will have completely automated primer, \\ basecoat and topcoat application. With this new plant, FAW-VW has expanded its \\ capacities at the Changchun site.
}

$D^{\text {is }}$ ürr is responsible for the construction of the complete paint shop for FAW-VW at the Changchun site, including application technology and exhaust air purification. In striving to ensure sustainable finishing, the car maker is relying on state-of-the-art Dürr technology. For this reason, the EcoDryScrubber has been put into use once again in Changchun. This system for the dry separation of overspray reduces energy consumption by up to 60 percent compared to conventional spray booths by using air recirculation. Fresh water and coagulation chemicals can be completely dispensed with and overspray bound by limestone powder can be reused.

\section{0 percent lower solvent emissions}

Another new and environmentally friendly feature of the plant is the use of water-based paints in the primer and basecoat section. Nevertheless, as with the clearcoat application, exhaust air flow from the paint booth is cleaned before it leaves the plant. This is a first in China, and a special feature worldwide. The EcoDryScrubber with its air recirculation and the highly efficient "Ecopure KPR" exhaust air purification system create a perfect symbiosis, since the use of dry separation in the spray booth produces highly concentrated and particularly efficiently filtered exhaust air. This allows for the use of a very compact exhaust air purification system and lowers the investment costs of the exhaust system by 60 percent. In this combina-

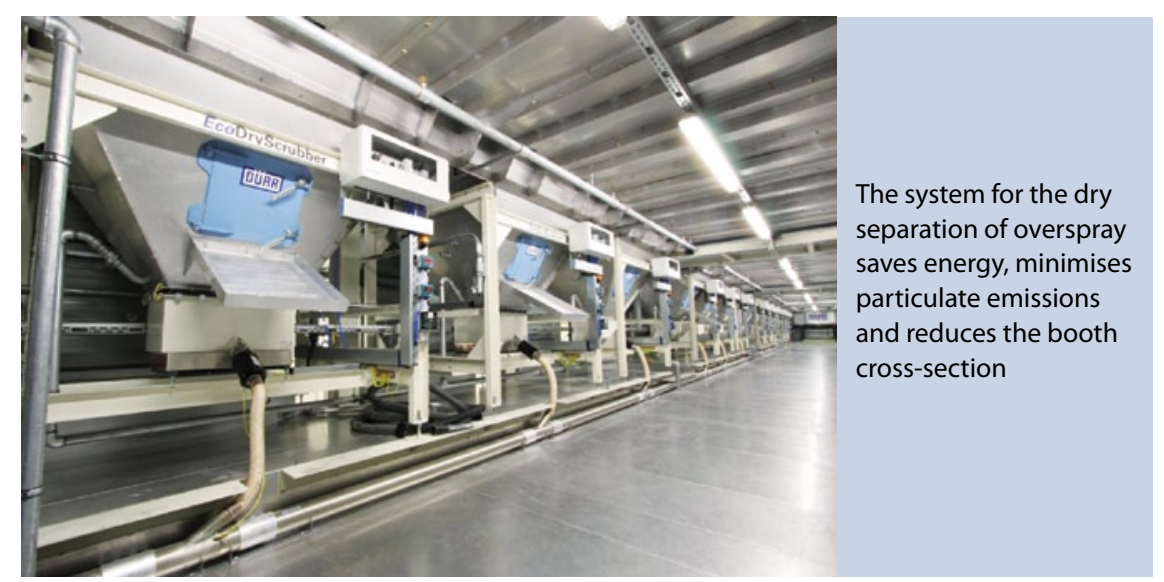

tion, the energy demand is reduced by 80 percent and the solvent emissions by as much as 90 percent. The exhaust air from the ovens is cleaned by the integrated "Ecopure TAR" afterburner, where the recovered heat is then in turn used to heat the ovens.

\section{Huge reduction in energy} and chemicals consumption

The RoDip M rotational dip coating process, which is already used around the world in 46 different paint shops for $\mathrm{PT} / \mathrm{ED}$ applications, is used in pretreatment and electro dipping in Changchun. In this process, the rotation of the entire body in the tank optimises the dipping, flooding and draining process. By eliminating the entry and exit ramps, the tank volume is decreased, thus dramatically reducing the need for energy and chemicals.

Prior to the filler and basecoat applications, the car body is cleaned by six EcoRS Clean robots and sword brushes.
Interior and exterior painting is fully automated, using a total of 62 painting and handling robots from the EcoRP $\mathrm{L}$ series.

EcoBell3, the new atomiser generation for electrostatic paint application with better performance and less complexity, provides the perfect application of primer, basecoat and clearcoat. Seam sealing, including the hemflange application, underbody protection and pore recognition, is carried out by 16 EcoRS robots.

This automotive paint shop, with the lowest emissions in China and a capacity of 34 vehicles per hour, will go into operation to paint various Audi models starting in March 2013. The contract, which Dürr is handling as general contractor, was awarded in the third quarter of 2011. I

\section{The Author:}

Günter Buzer, Dürr Systems GmbH,

Marketing Paint and Final Assembly Systems, Stuttgart, Germany, Tel. +49 7142 78-2614, guenter.buzer@durr.com,www.durr.com 